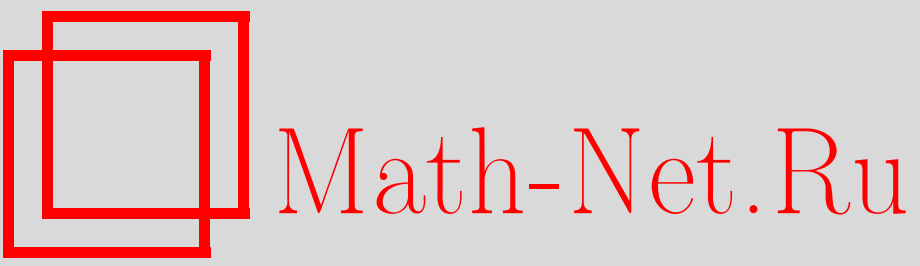

Л. А. Пальцев, О неравновесных процессах в умеренно плотном многоатомном газе во внешнем магнитном поле, ТМФ, 1996, том 107, номер 2, 307-319

DOI: https://doi.org/10.4213/tmf1157

Использование Общероссийского математического портала Math-Net.Ru подразумевает, что вы прочитали и согласны с пользовательским соглашением

http://www . mathnet.ru/rus/agreement

Параметры загрузки:

IP : 54.198 .55 .26

26 апреля 2023 г., 16:02:30 
ТЕОРЕТИЧЕСКАЯ

И МАТЕМАТИЧЕСКАЯ

ФИЗИКА

Том 107, № 2

май, 1996

\title{
Л. А. Пальцев \\ О НЕРАВНОВЕСНЫХ ПРОЦЕССАХ В УМЕРЕННО ПЛОТНОМ МНОГОАТОМНОМ ГАЗЕ ВО ВНЕШНЕМ МАГНИТНОМ ПОЛЕ
}

\begin{abstract}
Методами квантовой кинетической теории рассматриваются диссипативные и релаксационные процессы в умеренно плотном многоатомном газе во внешнем магнитном поле. В диссипативном приближении Навье-Стокса получены потоки импульса и энергии и коэффициенты переноса с учетом первой вириальной поправки. Найдена зависимость тензорного коэффициента сдвиговой вязкости в газе диамагнитных молекул от эффективных сечений парных и тройных столкновений и величины магнитного поля. Выводится уравнение Блоха для намагниченности в пространственно однородном газе линейных диамагнитных молекул. Получены времена релаксации и сдвиг ларморовой частоты за счет межмолекулярных столкновений.
\end{abstract}

Процессы переноса в многоатомных молекулярных системах во внешнем магнитном поле при любых значениях $\omega \tau$ ( $\omega$ - ларморова частота, $\tau$ - время свободного пробега молекул) подробно исследованы методами квантововй кинетической теории в разреженных газах и газах при нормальной плотности (см., например, [1-3] и библиографию в этих работах). В частности, было показано, что при $\omega \tau \simeq 1$ тензор напряжений и поток энергии зависят от взаимной ориентации магнитного поля и тензора скоростей деформации и градиента температуры; получены тензорные коэффициенты переноса, зависящие от эффективных сечений парных столкновений и величины магнитного поля. Это позволило не только объяснить эффекты Зенфтлебена-Беенаккера, Скотта и т.п., но и получить количественную информацию о величине эффективных сечений, существенно зависяших от структуры нецентральных межмолекулярных сил. Что касается умеренно плотных газов, то в работах $[4,5]$ рассматривались методами квантовой кинетической теории диссипативные и релаксационные процессы только при $\omega \tau \simeq \mu \ll 1$, где $\mu$ - параметр пространственной неоднородности газа. Здесь коэффициенты переноса и время релаксации плотности внутреннего момента импульса молекул (поляризуемость) не зависят от величины и направления магнитного поля.

В данной работе исследуются неравновесные процессы в умеренно плотном многоатомном газе во внешнем магнитном поле при $\omega \tau \simeq 1$. В частности, рассматриваются диссипативные процессы в пространственно неоднородных состояниях газа, близких к состоянию теплового равновесия, и релаксация поляризуемости и намагниченности в пространственно однородном газе. Получено линеаризованное кинетическое уравнение для оператора Вигнера, которое решается методом Боголюбова в газодинамическом приближении. В диссипативном приближении Навье-Стокса определены оператор Вигнера, потоки импульса и энергии и тензорные кинетические коэффициенты вязкости и теплопроводности с учетом первой вириальной поправки. Для газа линейных диама- 
гнитных молекул с малой несферичностью межмолекулярного взаимодействия получена зависимость тензорного коэффициента сдвиговой вязкости от величины магнитного поля и эффективных сечений парных и тройных столкновений. Показано, что в компонентах этого тензора в умеренно плотном газе присутствуют члены с иной, чем в газе при нормальной плотности, зависимостью от величины магнитного поля, что позволяет в принципе, измеряя вязкие силы, найти величину эффективных сечений тройных столкновений. Из нелинейного кинетического уравнения для пространственно однородного газа линейных диамагнитных молекул методом Боголюбова выводится уравнение Блоха для намагниченности и получены тензор обратных времен релаксации и сдвиг ларморовой частоты за счет межмолекулярных столкновений. В трехмоментном приближении найдена зависимость времен продольной и поперечной релаксаций и сдвига ларморовой частоты от эффективных сечений парных столкновений и величины магнитного поля.

1. В работе [4] получено кинетическое уравнение для умеренно плотного многоатомного газа, молекулы которого взаимодействуют с постоянным внешним магнитным полем $\mathbf{B}$, при $\omega \tau \simeq \mu$. В случае, когда $\omega \tau \simeq 1$, аналогично [4] получаем кинетическое уравнение

$$
\begin{aligned}
\frac{\partial}{\partial t} f_{1}+\mu & \frac{p_{1}^{j}}{M} \nabla_{j} f_{1}+\mu \varepsilon \sum_{k=1}^{2} N_{k}(1 ; f)+\varepsilon N_{3}(1 ; f)-\varepsilon J_{3}(1 ; f)= \\
= & J_{2}(1 ; f)-i\left[\Delta H(1) ; f_{1}\right] / \hbar
\end{aligned}
$$

здесь $f_{1}=U_{t} f\left(x_{1}\right) U_{-t}$, где $f\left(x_{1}\right)$ - одночастичный оператор Вигнера (см. обозначения к $\left.(4,1.1)^{1)}\right)$, определяется в пространстве собственных векторов $|1\rangle$ оператора $H_{1}$, соответствующих энергии $E_{1} ; H_{j}$ - часть гамильтониана $j$-й молекулы, соответствующая внутримолекулярным процессам с характерной частотой $\omega_{0} \simeq 1 / \tau_{0}$, где $\tau_{0}$ - время столкновения молекул; $U_{t}=\exp \left(i H_{1} t / \hbar\right) ; \Delta H(1)$ - диагональная в представлении функций $|1\rangle$ по квантовым числам, задающим энергию $E_{1}$, часть гамильтониана, соответствуюшая внутримолекулярным процессам с характерной частотой $\omega \simeq 1 / \tau$; в частности, в $\Delta H(1)$ включается энергия взаимодействия молекулы с внешним полем (см. [4]); $\mathbf{p}_{j}$ и $M$ - импульс центра масс и масса молекулы. Выражения для поправок на нелокальность $N_{k}(1 ; f)$, где $k=1,2$, и взаимодействие молекул с магнитным полем $N_{3}(1 ; f)$ к локальному интегралу парных столкновений, а также локальные интегралы парных $J_{2}(1 ; f)$ и тройных $J_{3}(1 ; f)$ столкновений даны в $(4, \Pi .3)$ и $(4, \Pi .1)$ (см. также $(П .5)$ в приложении). Параметр $\mu$ и параметр разреженности $\varepsilon$ поставлены в (1.1) только для указания порядка вклада соответствующих членов в изменение оператора $f_{1}$ за время $\tau$.

Ниже при рассмотрении диссипативных процессов ограничимся близкими к тепловому равновесию при температуре $T$ состояниями газа многоатомных молекул, у которых возбуждены только врашательные степени свободы. В равновесном состоянии решением кинетического уравнения (1.1) при дополнительных условиях $(4,2.6)$, в которых $n_{v}=n \delta_{v 0}$ (основное колебательное состояние), является оператор

$$
f_{1}(T)=f_{1 M}\{1+\varepsilon[n g(1)+2 n B-\Delta H(1) / T]\},
$$

где $f_{1 M}$ - оператор Максвелла-Больцмана, нормированный на плотность числа молекул $n$, в котором оператором внутренней энергии является $H_{1}$; одночастичный оператор $g(1)=\int d 2 X_{12} n f_{1 M}^{-1}$, где $\int d k \cdots=\operatorname{Tr}_{(k)} \int d \mathbf{p}_{k} \ldots, \operatorname{Tr}_{(i)}-$ след по состоянию $|i\rangle$;

\footnotetext{
1) Ссылки на формулы из работ $[4,5]$ обозначены следующим образом: $(4,1.1), \ldots,(5,1.1)$ и т.д.
} 
двухчастичный оператор $X_{12}$ определяется в $(4,2.2)$ (см. также $(П .3)$ при $\left.\varphi(j)=1 / 2\right)$; $B=-\int d 1 g(1) / 2$ - второй вириальный коэффициент.

В состояниях, близких к равновесному, будем искать оператор $f_{1}$ в виде

$$
f_{1}=f_{1}(T)+\rho_{1} \varphi(1) \rho_{1},
$$

где $\rho_{j}=\left[f_{j M}\right]^{1 / 2}$. Учитывая (1.2), для оператора $\varphi(1)$ из (1.1) в линейном приближении получаем уравнение

$$
\frac{\partial}{\partial t} \varphi(1)+\mu \nabla_{j}\left(p_{1}^{j} \varphi(1) / M+\varepsilon \sum_{k=1}^{2} I_{k}^{j} \varphi\right)+\varepsilon W(1 ; \varphi)=I_{B} \varphi,
$$

где

$$
W(1 ; \varphi)=I(B) \varphi-I\left\{\frac{\Delta H}{2 T} ; \varphi\right\}-2 n B I \varphi-I_{3} \varphi .
$$

В (1.3) супероператоры $I_{k}^{j}$ и $I(B)$ в поправках на нелокальность столкновений и взаимодействие молекул с магнитным полем к локальному интегралу парных столкновений определяются по формуле (П.1) при замене оператора $\varphi_{k E}$ операторами $\varphi_{k E}^{j}(P)$ и $\varphi_{3 E}(P)$, соответственно; супероператоры локальных парных $I, I_{B}$ и тройных $I_{3}$ столкновений определяются по формуле (П.3); здесь и в приложении $\{\ldots ; \ldots\}$-антикоммутатор. Отметим, что при получении (1.2) учли, что $N_{3}\left(1 ; f_{M}\right)=-\rho_{1} I_{B} \Delta H \rho_{1} / T$.

Из уравнения (1.3) аналогично [4] получаем уравнения переноса

$$
\begin{aligned}
& \frac{\partial}{\partial t} n^{*}=-\mu \nabla_{j} u^{j}, \\
& \frac{\partial}{\partial t} \mathcal{E}^{*}=-\mu \nabla_{j} q^{j} / n T, \\
& \frac{\partial}{\partial t} u^{k}=-\mu \nabla_{j} \pi^{k j} / n M,
\end{aligned}
$$

где локальные макроскопические переменные $n^{*}=\Delta n / n, \mathcal{E}^{*}=\Delta \mathcal{E} / n T$ ( $\Delta n$ и $\Delta \mathcal{E}$ отклонения плотности числа молекул и плотности энергии от их равновесных значений) и газодинамическая скорость $u^{k}$ имеют вид

$$
\begin{aligned}
& n^{*}=(1, \varphi), \\
& \mathcal{E}^{*}=(\varepsilon(p ; B), \varphi)+\left(\varepsilon_{T}(12) ; \Sigma \varphi\right), \\
& u^{k}=\left(p^{k}, \varphi\right) / M,
\end{aligned}
$$

поток энергии и тензор напряжений -

$$
\begin{aligned}
q^{j} & =n T\left(p^{j} \varepsilon(p ; B), \varphi\right) / M+n T\left(\varepsilon(p), I_{1}^{j} \varphi\right)+n T\left(P^{j} \varepsilon_{T}(12) ; \Sigma \varphi\right) / 2 M, \\
\pi^{k j} & =n\left(p^{k} p^{j}, \varphi\right) / M+n\left(p^{k}, I_{1}^{j} \varphi\right) .
\end{aligned}
$$

В (1.5) и (1.6) одночастичные и двухчастичные скалярные произведения равны

$$
\begin{aligned}
(a, \varphi) & =\int d 1 a^{\dagger}(1) \rho_{1} \varphi(1) \rho_{1}, \\
(a(12) ; \Sigma \varphi) & =\int d 1 d 2 a^{\dagger}(12) \rho_{12} \sum_{j=1}^{2} \varphi(j) \rho_{12} / n,
\end{aligned}
$$

где $\rho_{1 \ldots s}=\rho_{1} \ldots \rho_{s} ; \varepsilon_{1}(p ; B)=\varepsilon_{1}(p)+\Delta H(1) / T ; \varepsilon_{1}(p)=\left(p_{1}^{2} / 2 M+H_{1}\right) / T ; \varepsilon_{T}(12)=$ $\varepsilon_{\Phi}(12) / T ; \varepsilon_{\Phi}(12)$ - оператор энергии межмолекулярного взаимодействия (см. обозначения к $(4,1.4)) ; \mathbf{P}=\mathbf{P}_{1}+\mathbf{P}_{2}$. При выводе (1.4) было использовано выражение (П.2). 
2. Для исследования влияния внешнего магнитного поля на диссипативные процессы в умеренно плотном многоатомном газе и, в частности, на коэффициенты переноса рассмотрим решение кинетического уравнения (1.3) в газодинамическом приближении, когда $\mu \ll 1$. Следуя Боголюбову [6], будем искать $\varphi(1)$ в классе вигнеровских операторов, зависящих от времени через макроскопические переменные (1.5), в виде ряда

$$
\begin{aligned}
& \varphi(1)=\varphi_{1}^{(0)}+\mu \varphi_{1}^{(1)}+\mu^{2} \varphi_{1}^{(2)}+\ldots \\
& \varphi_{1}^{(j)}=\varphi_{1}^{(j ; 0)}+\varepsilon \varphi_{1}^{(j ; 1)}
\end{aligned}
$$

при следуюших дополнительных условиях: $n^{*}$ и $u^{k}$ полностью определяются операто$\operatorname{pom} \varphi_{1}^{(0,0)}$,

$$
\begin{gathered}
\left(\hat{\varepsilon}(p), \varphi^{(s ; k)}\right)+\left(\varepsilon_{T}(12) ; \Sigma \varphi^{(s ; k-1)}\right)= \\
=\delta_{s 0}\left\{\delta_{k 0} C_{V} T^{*}-n \delta_{k 1}\left(2 n^{*} / T+T^{*} T \frac{d}{d T}\right) T^{2} \frac{d B}{d T}\right\}, \\
s=0,1,2, \ldots, \quad k=0,1,
\end{gathered}
$$

здесь вместо энергии $\mathcal{E}^{*}$ ввели относительное отклонение температуры от ее равновесного значения $T^{*}=\Delta T / T$ по формуле

$$
\mathcal{E}^{*}=E_{n ; T} n^{*} / T+E_{T ; n} T^{*} / n,
$$

где частная производная $b_{a ; c}=(\partial b / \partial a)_{c}$;

$$
\begin{aligned}
E & =n T \mathcal{E}-n^{2} T^{2} \frac{d B}{d T}, & \mathcal{E} & =3 / 2+e / T, \\
e & =T^{2} d \ln Z / d T, & Z & =\operatorname{Tr}_{(1)} \exp \left(-H_{1} / T\right), \\
\hat{\varepsilon}_{1}(p) & =\varepsilon_{1}(p)-\mathcal{E}, & C_{V} & =d T \mathcal{E} / d T .
\end{aligned}
$$

Ниже, ссылаясь на условия (2.2), будем иметь в виду также условия для $n^{*}$ и $u^{j}$.

В локально-равновесном приближении из (1.3) с учетом (2.1) и (1.4) получаем уравнения

$$
W\left(1 ; \varphi^{(0 ; k-1)}\right)=I_{B} \varphi_{(0 ; k)}, \quad k=0,1,
$$

решением которых при условиях (2.2) являются операторы

$$
\begin{aligned}
\varphi_{1}^{(0 ; 0)}= & n^{*}+\hat{\varepsilon}_{1}(p) T^{*}+\mathbf{p}_{1} \mathbf{u} / T \\
\varphi_{1}^{(0 ; 1)}= & 2 n\left(\varphi_{1}^{(0 ; 0)}+n^{*}+T^{*} T \frac{d}{d T}\right) B-\Delta H(1)\left(\varphi_{1}^{(0 ; 0)}-T^{*}\right) / T+ \\
& +2 n^{*} g(1)+T^{*} T g_{T ; n \mathbf{p}}(1)+M \mathbf{u} g_{\mathbf{p} ; n T}(1) .
\end{aligned}
$$

При получении $(2.3)$ учли, что $I(B) \varphi^{(0 ; 0)}=I_{B} \Delta H T^{*} / T$.

В этом приближении имеем

$$
q^{(0) j}=(p+E) u^{j}, \quad \pi^{(0) i j}=\delta_{i j} n T\left(p_{n ; T} n^{*} / T+p_{T ; n} T^{*} / n\right),
$$

где $p=n T(1+n B)$. 
В диссипативном навье-стоксовском приближении для поправок к вигнеровским операторам (2.3), учитывая (1.4) и (2.4), получаем систему линейных неоднородных уравнений

$$
\begin{aligned}
a_{k}^{j}(1 ; B) \nabla_{j} T^{*}+ & b_{k}^{i j}(1 ; B)[\nabla u]^{i j}+c_{k}(1 ; B) \nabla_{j} u^{j}- \\
& -\delta_{k 1} \varepsilon^{i j l} R s^{i} \nabla_{j} u^{l} / 2 T=I_{B} \varphi^{(1 ; k)}, \quad k=0,1
\end{aligned}
$$

здесь $a_{0}^{j}(1 ; B)=a^{j}(1)$ и аналогично для тензорного и скалярного операторов, где

$$
\begin{aligned}
a^{j}(1) & =p_{1}^{j}\left(\hat{\varepsilon}_{1}(p)-1\right) / M, \\
b^{i j}(1) & =\left[p_{1} p_{1}\right]^{i j} / M T, \\
c(1) & =p_{1}^{2} / 3 M T-1-\hat{\varepsilon}_{1}(p) / C_{V},
\end{aligned}
$$

и поправки по плотности к этим операторам

$$
a_{1}^{j}(1 ; B)=\Delta a^{j}(1 ; 0)+\Delta a^{j}(1 ; B)
$$

где операторы в отсутствие магнитного поля $\Delta a^{j}(1 ; 0)=\Delta a_{v=0}^{j}(1)$ определяются в $\left(5\right.$, П.1.1) (основное колебательное состояние), $\Delta a^{j}(1 ; B)=-\hat{D}(B) a^{j}(1)$ и аналогично для тензорного и скалярного операторов. Супероператор имеет вид

$$
\hat{D}(B)=I(B) R_{B}^{-1}+R\left\{\frac{\Delta H}{2 T} ; R_{B}^{-1} \cdots\right\}+\left(R_{3}+2 n B R\right) R(B)^{-1}
$$

где $R(B)^{-1}=R_{B}^{-1}-R^{-1} ; R$ - супероператор Вальдмана-Снайдера [1]; $R_{B}=R+$ $i[\Delta H(1) ; \ldots] / \hbar$. Супероператор тройных столкновений $R_{3}$ определяется, как и $R$, в пространстве вигнеровских операторов $\psi(1)$, диагональных в представлении функций $|1\rangle$ по квантовым числам, задаюшим энергию $E_{1}$, по формуле $R_{3} \psi=-I_{3} \psi$. Неприводимый тензор второго ранга $[a c]^{i j}=\frac{1}{2}\left(a^{i} c^{j}+a^{j} c^{i}\right)-[a c] \delta_{i j} ;[a c]=a^{k} c^{k} / 3 ; \varepsilon^{i j k}$ - полностью антисимметричный единичный тензор третьего ранга. Заметим, что в (2.5) учтено, что $I_{2}^{j} p^{k} \varepsilon^{j k i}=-R s^{i}$, где $\mathbf{s}_{j}$ - оператор полного внутреннего момента импульса $j$-й молекулы.

Из (2.5) при условиях (2.2) находим при $k=2$ известньй в кинетике газа при нормальной плотности [1] оператор $\varphi_{1}^{(1 ; 0)}$ и поправку по плотности газа к этому оператору:

$$
\begin{aligned}
\varphi^{(1 ; 1)}= & \varphi^{(1 ; 1)}(1)-\hat{G}(B)\left(a^{j}(1) \nabla_{j} T^{*}+b^{i j}(1)[\nabla u]^{i j}+c(1) \nabla_{j} u^{j}\right)- \\
& -\varepsilon^{i j k} R(B)^{-1} R s^{i} \nabla_{j} u^{k} / 2 T+\hat{\varepsilon}_{1}(p)\left(\varepsilon_{T}(12) ; \Sigma R(B)^{-1} c\right) \nabla_{j} u^{j} / C_{V},
\end{aligned}
$$

здесь вигнеровский оператор при отсутствии магнитного поля $\varphi^{(1 ; 1)}(1)$ получаем из $(5,1.2)$ при $X_{12}^{(1)}=0, n_{v}=n \delta_{v 0}, \Delta A_{v}^{j}\left(x_{1}\right)=\delta_{v 0} \Delta A^{j}(1)$ и аналогично для тензорного и скалярного операторов; кроме того, $\nabla_{j} \ln T$ следует заменить на $\nabla_{j} T^{*}$. Супероператор $\hat{G}(B)$ определяется формулой

$$
\hat{G}(B) d=R(B)^{-1} \Delta d(0)-R_{B}^{-1} \hat{D}(B) d .
$$


Из (1.4) с учетом (2.6) находим в диссипативном навье-стоксовском приближении поток энергии и тензор напряжений:

$$
\begin{aligned}
q^{(1) j} & =q^{j}(0)+q^{j}(B), \\
\pi^{(1) i j} & =\pi^{i j}(0)+\pi^{i j}(B)+\delta_{i j} \pi(B),
\end{aligned}
$$

здесь поток энергии и тензор напряжений при отсутствии магнитного поля (cм. $(5,1.4)$ в основном колебательном состоянии) имеют вид

$$
\begin{aligned}
q(0) & =-\lambda T \nabla_{j} T^{*} \\
\pi^{i j}(0) & =-2 \eta_{1}[\nabla u]^{i j}-\delta_{i j} \eta_{2} \nabla_{k} u^{k}
\end{aligned}
$$

где $\lambda=\lambda_{0}+\varepsilon \Delta \lambda ; \quad \lambda_{0}-$ коэффициент теплопроводности в газе при нормальной плотности $[1,7]$ и поправка по плотности газа к этому коэффициенту $\Delta \lambda$ дается в $(5,1.5)$; аналогичное имеем и для коэффициентов сдвиговой $\eta_{1}$ и объемной $\eta_{2}$ вязкостей.

Поток энергии и тензор напряжений, обусловленные взаимодействием молекул с магнитным полем, принимают следуюший вид:

$$
\begin{aligned}
q^{j}(B) & =-\lambda^{j i}(B) T \nabla_{i} T^{*} \\
\pi^{i j}(B) & =-2 \eta_{1}(B)^{i j k l}[\nabla u]^{k l}-\eta_{12}(B)^{i j} \nabla_{k} u^{k} \\
\pi(B) & =-\eta_{21}(B)^{k l}[\nabla u]^{k l}-\eta_{2}(B) \nabla_{k} u^{k}
\end{aligned}
$$

здесь $\lambda^{j i}(B)=\lambda_{0}^{j i}(B)+\varepsilon \Delta \lambda^{j i}(B)$, где $\lambda_{0}^{j i}(B)$ - известная [1] поправка к $\lambda_{0}$,

$$
\Delta \lambda^{j i}(B)=n\left(a^{j}, \hat{G}(B) a^{i}\right)+n\left(\varepsilon(p), g^{j}(B) a^{i}\right)+n\left(P^{j} \varepsilon_{T}(12) ; \Sigma R(B)^{-1} a^{i}\right) / 2 M,
$$

где $g^{j}(B) d=I_{1}^{j} R(B)^{-1} d$. Аналогичное имеем и для коэффициентов переноса в тензоре напряжений, где

$$
\begin{aligned}
2 \Delta \eta_{1}(B)^{i j k l} & =n T\left(b^{i j}, \hat{G}(B) b^{k l}\right)+n\left[\left(p, g(B) b^{k l}\right)\right]^{i j}, \\
\Delta \eta_{12}(B)^{i j} & =n T\left(b^{i j}, \hat{G}(B) c\right)+n[(p, g(B) c)]^{i j}, \\
\Delta \eta_{21}(B)^{i j} & =n T\left(c, \hat{G}(B) b^{i j}\right)+n\left[\left(p, g(B) b^{i j}\right)\right], \\
\Delta \eta_{2}(B) & =n T(c, \hat{G}(B) c)+n[(p, g(B) c)]-n T\left(\varepsilon_{T}(12) ; \Sigma R(B)^{-1} c\right) / C_{V} .
\end{aligned}
$$

Заметим, что в настояшее время реализовать условие $\omega \tau \simeq 1$ в умеренно плотном газе возможно только для парамагнитных молекул, поскольку для диамагнитных молекул, например для $\mathrm{HD}$, при нормальной температуре необходимо поле $B \simeq 30 p$ T, где $p$ - давление, измеряемое в атмосферах. Однако в качестве примера найдем зависимость от величины и направления магнитного поля кинетических коэффициентов в тензоре сдвиговых напряжений в газе линейных диамагнитных молекул с малой несферичностью межмолекулярного взаимодействия. В этом случае $H_{1}=\hbar^{2} s_{1}^{2} / 2 I_{0}$ и $\Delta H(1)=-\hbar \omega s_{1 z}$, где здесь и везде ниже $\hbar \mathbf{s}_{j}$ и $\hbar s_{j z}$ - операторы полного внутреннего момента импульса $j$-й молекулы и его проекции на направление внешнего магнитного поля; $I_{0}$ - момент инерции молекулы; $\omega=g \mu_{n} B / \hbar ; \mu_{n}$ - ядерный магнетон; $g$-фактор не зависит от внутреннего состояния молекулы. Относительная величина вклада нецентральных межмолекулярных сил в интегралах столкновений и поправках к этим интегралам характеризуется параметром несферичности $\varkappa[1,4]$, который будем считать малым. 
Ограничимся, как и в газе при нормальной плотности [1], двухмоментным приближением, когда в разложении оператора $R_{B}^{-1} b^{i j}$ по неприводимым тензорным операторам $[7,8]$ учитываются только члены, содержашие ортонормированные тензоры второго ранга $\boldsymbol{\Phi}^{20}(1)=\left[\mathbf{p}_{1} \mathbf{p}_{1}\right] / M T(2)^{1 / 2}$ и $\boldsymbol{\Phi}^{02}(1)=\left(15 / 2\left\langle s^{2}\left(s^{2}-3 / 4\right)\right\rangle\right)^{1 / 2}\left[\mathbf{s}_{1} \mathbf{s}_{1}\right]$, где $\langle\ldots\rangle=\operatorname{Tr}_{(1)} \exp \left(-H_{1} / T\right) \ldots$ Тогда из $(2.7)$ с точностью до членов второго порядка по $\varkappa$ для тензора сдвиговых напряжений в представлении сферических тензоров [9] получаем следуюшее выражение:

$$
\pi(B)_{2 m}=-2 \eta_{m}(B)[\nabla u]_{2 m}
$$

здесь кинетический коэффициент $\eta_{m}(B)=\eta_{m}^{(0)}(B)+\varepsilon \eta_{m}^{(1)}(B)$, где известная в кинетике газа при нормальной плотности [1] поправка к коэффициенту сдвиговой вязкости $\eta$ обусловлена взаимодействием молекул с магнитным полем:

Поправка по плотности газа к $\eta_{m}^{(0)}(B)$ равна

$$
\eta_{m}^{(0)}(B) / \eta=-\psi(02) F\left(m \xi_{02}\right) .
$$

$$
\begin{aligned}
\eta_{m}^{(1)}(B) / \eta= & -\psi(02) \alpha(02) F\left(m \xi_{02}\right)-\psi(02)\left\{\sigma_{23}(02)-n v \sigma(02) \times\right. \\
& \left.\times \int_{0}^{\infty} d \tau \exp \left(-\eta^{*} \tau\right)[1-\sigma(\tau ; 02)]\right\} F^{2}\left(m \xi_{02}\right), \quad \eta^{*} \rightarrow 0^{+}
\end{aligned}
$$

где

$$
\begin{aligned}
\psi(02) & =\left|\sigma^{(1)}\left(\begin{array}{ll}
0 & 2 \\
2 & 0
\end{array}\right)\right|^{2} / \sigma(02) \sigma(20), \\
F(x) & =f^{(+)}(x)+i f^{(-)}(x)=x(x+i) /\left(1+x^{2}\right), \\
\xi_{(02)} & =\omega / n v \sigma(02) .
\end{aligned}
$$

Эффективные сечения парных столкновений в (2.8) [8] определяются формулой

$$
\sigma^{(k)}\left(\begin{array}{cc}
i^{\prime} & j^{\prime} \\
i & j
\end{array}\right)=\left(\Phi_{2 m}^{i^{\prime} j^{\prime}}, R^{(k)} \Phi_{2 m}^{i j}\right) / n v
$$

и не зависят от $m$, где $v=(16 T / \pi M)^{1 / 2} ; R^{(0)}$ - супероператор Вальдмана-Снайдера для "центральных" межмолекулярных сил $[1,4] ; R^{(1)}=R-R^{(0)}$. Аналогичным образом ниже определяются операторы и супероператоры. Относительные эффективные сечения тройных $\sigma_{23}^{(k)}\left(\begin{array}{cc}i^{\prime} & j^{\prime} \\ i & j\end{array}\right)$ и парных $\sigma^{(k)}\left(\tau ; \begin{array}{cc}i^{\prime} & j^{\prime} \\ i & j\end{array}\right)$ столкновений находятся по формуле $(2.9)$, где $R^{(k)}$ следует заменить супероператорами $\left(R_{3}^{(k)}+2 n B R^{(k)}\right) / \sigma^{(k)}\left(\begin{array}{c}i^{\prime} j^{\prime} \\ i\end{array}\right)$ и $R^{(k)}(\tau) / \sigma^{(k)}\left(\begin{array}{cc}i^{\prime} & j^{\prime} \\ i & j\end{array}\right)$, соответственно. Супероператор $R(\tau)$ получаем из $R$ заменой операторов рассеяния Меллера $\Omega^{(+)}(12)$ [10] операторами эволюции $U(\tau)$ (см. обозначения к $(\Pi .1)) ; \sigma(i j)=\sigma^{(0)}\left(\begin{array}{c}i j \\ i j\end{array}\right)$ и аналогично для $\sigma_{23}(i j)$ и $\sigma(\tau ; i j)$. Кроме того, в $(2.8)$ имеем

$$
\begin{aligned}
& \alpha(02)=\sigma_{23}^{(1)}\left(\begin{array}{ll}
2 & 0 \\
0 & 2
\end{array}\right)-\sigma_{23}(20)-\sigma_{23}(02)+(1 / 50)^{1 / 2} \sum_{M}\left\{\left(\Phi_{2 M}^{20}, \Delta b^{(0)}(0)_{2 M}\right)-\right. \\
& -\left(\Phi_{2 M}^{(02)}, \Delta b^{(1)}(0)_{2 M}\right) \sigma(20) / \sigma^{(1)}\left(\begin{array}{cc}
0 & 2 \\
2 & 0
\end{array}\right)+\sum_{m, s}\langle 1 m 1 s \mid 2-M\rangle\left[\left(p_{1 m}, I_{1 ; 1 s}^{(0)} \Phi_{2-m}^{20}\right)-\right. \\
& \left.\left.-\left(p_{1 m}, I_{1 ; 1 s}^{(1)} \Phi_{2-M}^{02}\right) \sigma(20) / \sigma^{(1)}\left(\begin{array}{cc}
2 & 0 \\
0 & 2
\end{array}\right)\right]\right\}-n v \sigma(02) \int_{0}^{\infty} d \tau \exp \left(-\eta^{*} \tau\right)\left(\Sigma\left(\tau ; \begin{array}{ll}
2 & 2 \\
2 & 0
\end{array}\right)+\right. \\
& \left.+\Sigma\left(\tau ; \begin{array}{ll}
2 & 0 \\
0 & 2
\end{array}\right)+\sigma^{(1)}\left(\tau ; \begin{array}{ll}
2 & 0 \\
0 & 2
\end{array}\right)-\sigma(\tau ; 02)\right), \quad \eta^{*} \rightarrow 0^{+},
\end{aligned}
$$


где $\left\langle j_{1} m_{1} j_{2} m_{2} \mid J M\right\rangle$ - коэффициент Клебша-Гордана [9]. Относительные эффективные сечения $\Sigma\left(\tau ; \begin{array}{cc}i^{\prime} & j^{\prime} \\ i & j\end{array}\right)$ получаем по формуле (2.9), где $R$ следует заменить супероператором $\hat{S}(\tau)$, определяемым по формуле

$$
\hat{S}(\tau) \varphi=(i / \hbar) \int d 2\left\{\left[\Delta T(\tau) \Phi_{0}^{(1)}(P) \Omega^{\dagger}-T \Phi_{0}^{(1)}(P) \Delta \Omega(\tau)^{\dagger}\right]_{p p}^{(10)}-\text { э.c. }\right\},
$$

где $\Delta T(\tau)=\Phi(12) \Delta \Omega(\tau) ; \Delta \Omega(\tau)=\Omega_{12}-U(\tau) U^{(0)}(\tau)^{\dagger} \Omega_{12}^{(0)}$.

Из (2.8) следует, что в умеренно плотном газе диамагнитных молекул в коэффициенте сдвиговой вязкости присутствуют члены с иной, чем в газе при нормальной плотности, зависимостью от величины магнитного поля $\left(\sim F^{2}\left(m \xi_{02}\right)\right)$. Это позволяет в принципе по измерениям вязких сил найти эффективное сечение тройных столкновений $\sigma_{23}(02)$.

3. Как следует из (1.2), (2.2) и (2.6) в умеренно плотном многоатомном газе, молекулы которого взаимодействуют с внешним магнитным полем, возникает отличная от нуля поляризуемость $S^{j}=\int d 1 s_{1}^{j} f_{1}$. Для газа линейных диамагнитных молекул при магнитном поле, направленном вдоль оси $z$ (ось 3 ), $S^{j}$ равна $S_{B} \delta_{j 3}$ в равновесном состоянии, $\left(n^{*}+T^{*} T \frac{d}{d T}\right) S_{B} \delta_{j 3}$ в локально-равновесном приближении и $I^{j i}(\operatorname{rot} \mathbf{u})^{i} / 2$ в диссипативном приближении. Здесь $S_{B}=I \omega$, где $I=n \hbar / T N(s)^{2} ; N(s)=\left(\left\langle s^{2}\right\rangle / 3\right)^{1 / 2} ; I^{j j}=\left(I_{1}+\right.$ $\left.I_{-1}\right) / 2 ; \quad I^{j 3}=I^{3 j}=0, I^{12}=-I^{21}=i\left(I_{1}-I_{-1}\right) / 2, I_{m}=I\left(\Phi_{1-m}^{01}, R_{B}^{-1} R \Phi_{1-m}^{01}\right)$, ортонормированный векторный оператор $\boldsymbol{\Phi}^{01}(1)=\mathbf{s}_{1} N(s)$. Соответственно имеем и отличную от нуля намагниченность $M^{j}=g \mu_{n} S^{j}$.

В работе [5] рассмотрена релаксация поляризуемости в пространственно неоднородном газе к $I \operatorname{rot} \mathbf{u} / 2$ в случае, если $\omega \tau \simeq \mu \ll 1$ и $\simeq \simeq \mu$. Здесь рассмотрим релаксацию поляризуемости и намагниченности только в пространственно однородном умеренно плотном газе линейных диамагнитных молекул при $\omega \tau \simeq 1$ и $\varkappa 1$. Как и в [4], представим локальные интегралы парных и тройных столкновений и поправку к локальному интегралу парных столкновений за счет взаимодействия молекул с магнитным полем в виде суммы операторов, определяющих вклад центральных (индекс 0) и нецентральных (индекс 1) межмолекулярных сил. Аналогичным образом везде ниже будем определять функции, операторы и супероператоры. Тогда из (1.1) получаем уравнение

$$
\frac{\partial}{\partial t} f_{1}-i \omega\left[s ; f_{1}\right]=\sum_{k=0}^{1} \varkappa^{k} J^{(k)}(1 ; f)
$$

где

$$
J^{(k)}(1 ; f)=\sum_{j=0}^{1} \varepsilon^{j} J_{2+j}^{(k)}(1 ; f)-\varepsilon N_{3}^{(k)}(1 ; f) ;
$$

$s=s_{1 z}$ и параметры $\varkappa$ и $\varepsilon$ поставлены только для указания порядка вклада соответствуюших членов в изменение $f_{1}$ за время свободного пробега.

Из (3.1) получаем уравнение для поляризуемости

$$
\frac{\partial}{\partial t} S_{1 m}=-i \omega m S_{1 m}+\varkappa I_{1 m}(f),
$$

где

$$
I_{1 m}(f)=\int d 1 s_{1 m} J^{(1)}(1 ; f)
$$


и использованы сферические тензоры [9].

При $\varkappa \ll 1$ из $(3.2)$ следует, что $S_{1 m}^{(*)}=S_{1 m} \exp (i \omega m t)$ является медленно изменяющейся функцией за время $\tau$. Тогда для определения источника в уравнении (3.2) будем, следуя Боголюбову [6], искать вигнеровский оператор $f_{j m}=f_{j} \exp (i \omega m t)$ в классе операторов, зависящих от времени через $S_{1 m}^{(*)}$, в виде ряда

$$
\begin{aligned}
f_{1 m} & =f_{1 m}(0)+\varkappa f_{1 m}(1)+\varkappa^{2} f_{1 m}(2)+\ldots, \\
f_{1 m}(k) & =f_{1 m}(k ; 0)+\varepsilon f_{1 m}(k ; 1),
\end{aligned}
$$

где поляризуемость подчиняется уравнению

$$
\frac{\partial}{\partial t} S_{1 m}^{(*)}=\varkappa I_{1 m}^{(1)}+\varkappa^{2} I_{1 m}^{(2)}+\ldots
$$

Функции $I_{1 m}^{(k)}=\sum_{j=0}^{1} \varepsilon^{j} I_{1 m}^{(k ; j)}$ получаем обычным образом [6] из источника в уравнении (3.2), учитывая (3.3). В качестве дополнительных условий для однозначного определения операторов в (3.3) используем следующие условия: $n$ полностью определяется оператором $f_{10}(0 ; 0)$, поляризуемость $S_{1 m}^{(*)}$ - оператором $f_{1 m}(0 ; 1)$, а из определения энергии получаем

$$
\begin{gathered}
\int d 1 \varepsilon_{1}(p) f_{10}(s ; k)+\sum_{j=0}^{1} \int d 1 d 2 \varepsilon_{T}^{(j)}(12) \sum_{i=0}^{s-1} f_{10}(s-i-j ; k-1) f_{20}(i ; k-1)= \\
=n \mathcal{E} \delta_{s 0} \delta_{k 0}-\sum_{j=0}^{1} n^{2} T^{2} \frac{d B^{(j)}}{d T} \delta_{k 1} \delta_{s j}, \quad s=0,1,2, \ldots, \quad k=0,1 .
\end{gathered}
$$

В нулевом приближении из (3.1) имеем уравнение

$$
\exp (i \omega m t) J_{2}^{(0)}\left(1 ; F_{0}\right)+i \omega\left[s ; F_{m}\right]+i \omega m F_{m}=0,
$$

где $F_{m}=f_{1 m}(0 ; 0)$, решением которого при условиях (3.5) является оператор $f_{1 m}(0 ; 0)=\delta_{m 0} f_{1 m}$. Введем операторы $\varphi_{j m}(s ; k)=\rho_{j}^{-1} f_{j m}(s ; k) \rho_{j}^{-1}$. Тогда из $(3.1)$ получаем уравнение

$$
I_{B m} \varphi_{m}(0 ; 1)=\delta_{m 0} n I^{(0)} g^{(0)},
$$

где $I_{B m}=I^{(0)}+i \omega[s ; \ldots]+i \omega m$. Отметим, что $I_{B m} s_{1-m}=0$. Решением этого уравнения при условиях (3.5) является оператор

$$
\varphi_{1 m}(0 ; 1)=\delta_{m 0} n\left(2 B^{(0)}+g^{(0)}(1)\right)-(-1)^{m} S_{1 m}^{(*)} \Phi^{01}(1)_{1-m} N(s) / n,
$$

и, следовательно, источник в уравнении (3.4) имеет вид

$$
I_{1 m}^{(1)}=-\Delta S_{1 m} / \tau_{\varkappa}
$$

здесь $\Delta S_{1 m}=S_{1 m}^{(*)}-i \delta_{m 0} S_{B}$; время врашательной релаксации $\tau_{\varkappa}=1 / n v \sigma(01)[5]$, где эффективное сечение равно

$$
\begin{aligned}
\sigma(01)= & 8 n \pi^{4} \hbar^{2} \int d \mathbf{p} \sum_{E} \exp \left[-\left(p^{2} / m+E\right) / T\right] \times \\
& \times\left[\left[\mathbf{S} ; T_{12}\right] \delta\left(\frac{p^{2}}{M}+E-K\right)\left[\mathbf{S} ; T_{12}\right]^{\dagger}\right]_{p p}^{10} / 3 v Q\left\langle s^{2}\right\rangle,
\end{aligned}
$$


$\mathbf{S}=\mathbf{s}_{1}+\mathbf{s}_{2}, Q=(\pi M T)^{3 / 2} Z^{2}$ и использованы обозначения из приложения.

Учитывая (3.6), (3.2), (3.7), а также то, что $N_{3}^{(1)}\left(1 ; f_{M}\right)=\hbar \omega \rho_{1} I^{(1)} s \rho_{1} / T$, из (3.1) для оператора $\varphi_{1 m}(1 ; k)$ получаем уравнение

$$
\begin{aligned}
I_{B m} \varphi_{m}(1 ; k)= & \delta_{k 1}\left\{\delta_{m 0} n I^{(0)} g^{(1)}-I_{3}^{(0)} \varphi_{m}(1 ; k-1)\right\}- \\
& -(-1)^{m} \Delta S_{1 m} \xi(1)_{1-m} N(s) / n, \quad k=0,1,
\end{aligned}
$$

здесь аксиальньй векторный оператор $\boldsymbol{\xi}(1)=R^{(1)} \boldsymbol{\Phi}^{01}-\boldsymbol{\Phi}^{01}(1) / \tau_{\varkappa}$.

Из (3.8) при условиях (3.5) находим

$$
\varphi_{1 m}(1 ; k)=\delta_{k 1}\left\{\delta_{m 0} n\left(2 B^{(1)}+g^{(1)}(1)\right)+(-1)^{m} R_{B m}^{-1} \xi_{1-m} \Delta S_{1 m} N(s) / n\right\},
$$

где $R_{B m}=R_{B}^{(0)}-i \omega m, R_{B}^{(0)}=R^{(0)}-i \omega[s ; \ldots]$.

Подставляя (3.9) в выражение для источника в уравнении (3.4), получаем

$$
I_{1 m}^{(2)}=\left(\alpha_{m}(B) / \tau_{\varkappa}+i \omega m \beta_{m}(B)\right) \Delta S_{1 m},
$$

здесь

$$
\begin{aligned}
\alpha_{m}(B) / \tau_{\varkappa} & =\left(\Theta \xi_{1 m}, R_{B}^{(0)-1} \xi_{1-m}\right)-m \omega \operatorname{Im} \psi_{m}(B), \\
\beta_{m}(B) & =\operatorname{Re} \psi_{m}(B), \\
\psi_{m}(B) & =\left(\Theta \xi_{1 m}, R_{B m}^{-1} R_{B}^{(0)-1} \xi_{1-m}\right)
\end{aligned}
$$

где $\Theta$ - антиунитарный супероператор обрашения времени [1].

Из (3.4), учитывая (3.7) и (3.10), с точностью до членов второго порядка по $\varkappa$ получаем уравнение Блоха для намагниченности в умеренно плотном газе линейных диамагнитных молекул:

$$
\frac{\partial}{\partial t} M^{j}=\left(M_{B} \delta_{j 3}-M^{j}\right) / \tau_{j}+\varepsilon^{j k 3}(\omega+\Delta \omega(B)) M^{k},
$$

здесь $M_{B}=g \mu_{n} S_{B} ; \tau_{3}=\tau_{\|} ; \tau_{1}=\tau_{2}=\tau_{\perp}$. Времена продольной и поперечной релаксаций и сдвиг ларморовой частоты за счет межмолекулярных столкновений равны

$$
\begin{aligned}
\tau_{\|} & =\tau_{\varkappa}\left(1-\alpha_{0}(B)\right), \\
\tau_{\perp} & =\tau_{\varkappa}\left(1-\alpha_{1}(B)\right), \\
\Delta \omega(B) & =\omega \beta_{1}(B) .
\end{aligned}
$$

Функции $\alpha_{k}(B)$ и $\beta_{1}(B)$ можно найти известными методами квантовой кинетической теории газов $[1,8]$, представляя $\xi(1) 1 m$ в виде разложения по неприводимым тензорным операторам. Ограничимся при вычислении $\xi(1)_{1 m}$ и матричных элементов супероператоров тремя неприводимыми компонентами тензорного оператора третьего ранга:

$$
\Phi^{21}(1)_{l m}=\sum_{m_{1} m_{2}}\left\langle 2 m_{1} 1 m_{2} \mid l m\right\rangle \Phi^{20}(1)_{2 m_{1}} \Phi^{01}(1)_{1 m_{2}} .
$$


Тогда в приближении “сферической симметрии” [8], когда эффективные сечения $\sigma(21)_{l}$, определяемые, как и во втором разделе (см. (2.9)), через операторы $\Phi^{21}(1)_{l m}$ и супероператор Вальдмана-Снайдера $R^{(0)}$, равны $\sigma(21)$ при $l=1,2,3$, находим

$$
\begin{aligned}
\xi(1)_{1 m} & =n v \sigma^{(1)}\left(\begin{array}{ll}
2 & 1 \\
0 & 1
\end{array}\right) \Phi^{21}(1)_{1 m}, \\
\alpha_{k}(B) & =\psi(21)\left(1-3 F_{k}\left(\xi_{21}\right) / 5\right), \\
\beta_{1}(B) & =-3 \sigma(01) \psi(21)\left(1-2\left[F_{1}\left(\xi_{21}\right)+F_{0}\left(2 \xi_{21}\right)\right] / 5\right) / 2 \sigma(21),
\end{aligned}
$$

где $\sigma^{(1)}\left(\begin{array}{ll}2 & 1 \\ 0 & 1\end{array}\right)$ определяется по формуле $(2.9)$ через операторы $\Phi^{21}(1)_{1 m}, \quad \Phi^{01}(1)_{1 m}$ и эрмитову часть супероператора Вальдмана-Снайдера $R^{(1)}$;

$$
\begin{aligned}
\psi(21) & =\left|\sigma^{(1)}\left(\begin{array}{ll}
2 & 1 \\
0 & 1
\end{array}\right)\right|^{2} / \sigma(21) \sigma(01), \\
F_{k}(x) & =\left(1-\delta_{k 1} / 2\right) f^{(+)}(x)+\delta_{k 1} f^{(+)}(2 x), \\
\xi_{21} & =\omega / n v \sigma(21) .
\end{aligned}
$$

Отметим, что полученные выше времена продольной и поперечной релаксации зависят от величины магнитного поля аналогично зависимости коэффициентов теплопроводности $\lambda_{\|}$и $\lambda_{\perp}$ в э ффекте Зенфолебена-Беенаккера, где относительную частоту $\xi_{12}[1]$ следует заменить на $\xi_{21}$.

\section{Приложение}

Супероператоры в (1.3) определяются по формуле

$$
I_{k} \varphi=\frac{i}{4 \hbar} \rho_{1}^{-1} \int d 2\left(\sum_{E}\left[\varphi_{k E}\right]_{p p}^{(1 E)}-\text { э.c. }\right) \rho_{1}^{(-1)},
$$

где

$$
\begin{aligned}
\varphi_{k E}^{j}(P)= & T_{k}^{j} \Phi_{E}^{(k)}(P) \Omega_{12}^{\dagger}+T_{12} \Phi_{E}^{(k)} \Omega_{k}^{j \dagger} \\
\varphi_{3 E}(P)= & 4 T_{12}\left(\frac { i } { \hbar } \int _ { 0 } ^ { \infty } d \tau \operatorname { e x p } ( - \eta \tau ) \left(\left[\Phi_{E}^{(1)}(P) ; \Omega^{\dagger}(\tau) \Delta H \Omega(\tau)\right]-\right.\right. \\
& \left.\left.\quad-\Omega^{\dagger}(\tau)\left[\Phi_{E}^{(1)}(P) ; \Delta H\right] \Omega(\tau)\right)-\left\{\Delta H ; \Phi_{E}^{(1)}(P)\right\} / 2\right) \Omega_{12}, \quad \eta \rightarrow 0^{+},
\end{aligned}
$$

здесь, кроме обозначений из приложения в работе [4], введены следующие обозначения:

$$
\left[A_{1} \ldots s\right]_{p p^{\prime}}^{(j E)}=\sum_{E_{1} E_{2}} \Delta\left(E-E_{1}+E_{2}\right) P_{s E_{1}}^{(j)}(p) A_{1 \ldots s}(P) P_{s E}^{(j)}\left(p^{\prime}\right)^{\dagger}
$$

где $P_{s E}^{(1)}(p)=P_{s E}(p)$ и $P_{s E}^{(2)}(p)=P_{s E}(p)^{\dagger}($ см. обозначения к $(4, \Pi .2)) ; T_{1}^{j}=x^{j} T_{12}$, $T_{2}^{j}=T_{12} x^{j}$ и аналогично для $\Omega_{k}^{j}$, где $\mathbf{x}$ - оператор относительной координаты второй и первой молекул; $\Delta H=\sum_{j=1}^{2} \Delta H(j)$;

$$
\begin{gathered}
\Phi_{E}^{(k)}(P)=\int d \tau_{12}\left[\rho_{12}^{\prime}\left(\varphi\left(2^{\prime}\right)-(-1)^{k} \varphi\left(1^{\prime}\right)\right) \rho_{12}^{\prime}\right]_{p^{\prime} p^{\prime}}^{(2 E)} \\
\Omega(\tau)=U(\tau)^{\dagger} \Omega_{12} ; \quad U(\tau)=\exp (-i H \tau / \hbar) \exp (i K \tau / \hbar)
\end{gathered}
$$


$K$ и $H=K+\Phi(12)$ - операторы кинетической (поступательной и внутренней) и полной энергий первой и второй молекул в системе их центра масс.

Из (П.1) следует равенство

$$
(\varepsilon(p), I(B) \varphi)=(\Delta H, I \varphi) / T-i\left(\varepsilon_{T}(12) ; \Sigma[\Delta H ; \varphi]\right) / \hbar .
$$

Линейные супероператоры парных и тройных столкновений следующие:

$$
\begin{gathered}
I \varphi=\rho_{1}^{-1} \int d 2 I_{12} \varphi \rho_{1}^{-1} \\
I_{B} \varphi=I \varphi-i[\Delta H(1) ; \varphi(1)] / \hbar \\
I_{3} \varphi=\rho_{1}^{-1} \int d 2\left\{\frac{1}{2} \int d 3\left(I_{123} \varphi-(2 \pi \hbar)^{3} \delta^{(3)}(0) \sum_{j=2}^{3} f_{5-j M} I_{1 j} \varphi\right)-\sum_{j=1}^{2} I_{12}^{(j)} \varphi\right\} \rho_{1}^{-1}, \\
I_{1 \ldots s} \varphi=\sum_{E} \int d \tau_{1 \ldots s}^{(+)}(E)\left(T_{s E} \Phi_{1 \ldots s}\left(p^{\prime}\right) T_{s E}^{\dagger}-\left\{T_{s E} T_{s E}^{\dagger} ; \Phi_{1 \ldots s}(p)\right\} / 2\right)+ \\
+i \pi(3 \pi \hbar)^{3}\left[\Phi_{1 \ldots s}(p) ;\left(T_{s 0}+T_{s 0}^{\dagger}\right)\right] \\
\Phi_{1 \ldots s}(p)=\rho_{1 \ldots s} \sum_{j=1}^{s} \varphi(j) \rho_{1 \ldots s}
\end{gathered}
$$

$T_{s E}=\left[T_{1 \ldots s}\right]_{p p^{\prime}}^{(1 E)} ; T_{s 0}$ есть $T_{s E}$ при $E=0$ и $\mathbf{p}_{s j}=\mathbf{p}_{s j}^{\prime}$, где (здесь и в приложении работы [4]) относительный импульс $\mathbf{p}_{s j}=\mathbf{P}_{s} / s-\mathbf{p}_{j}(j=1, \ldots, s-1) ; I_{12}^{(j)} \varphi$ получаем из $I_{12} \varphi$ заменой $\Phi_{12}(p)$ оператором $n f_{j M} f_{3-j M} g_{j}(\varphi)$, где

$$
\begin{aligned}
g_{j}(\varphi) & =\int d 3 X_{j 3}(\varphi) / n f_{j M}, \\
X_{j k}(\varphi) & =\sum_{E} \int d \tau_{j k} \Omega_{2 E}(j k) \Phi_{j k}\left(p^{\prime}\right) \Omega_{2 E}(j k)^{\dagger}-(2 \pi \hbar)^{3} \delta^{(3)}(0) \Phi_{j k}(p),
\end{aligned}
$$

здесь $\Omega_{2 E}(j 3)$ определяется аналогично $T_{2 E}(j 3)$ через оператор рассеяния Меллера $\Omega_{j 3}^{(+)}$ [10].

Оператор

$$
N_{3}(1 ; f)=\int d 2\left(\sum_{E}\left[T_{12} f_{E} \Omega_{12}^{\dagger}\right]_{p p}^{(1 E)}+\text { ə.c. }\right)
$$

где

$$
\begin{gathered}
f_{E}=\int_{0}^{\infty} d \tau \exp (-\eta \tau)\left(\left[\Omega^{\dagger}(\tau) \Delta H \Omega(\tau) ; F_{2 E}(P)\right]-\right. \\
\left.\Omega^{\dagger}(\tau)\left[\Delta H ; F_{2 E}(P)\right] \Omega(\tau)\right), \quad \eta \rightarrow 0^{+}, \\
F_{2 E}(P)=(2 \pi)^{3} \hbar \int d \mathbf{p}\left[f_{1} f_{2}\right]_{p p}^{(2 E)},
\end{gathered}
$$

учитывает взаимодействие молекул с магнитным полем в процессе их столкновений. 


\section{Список литературы}

[1] Moraal H. // Phys. Rep. 1975. V. 17C. № 5. P. 225-306.

[2] Masur E., Beenakker J. J. M., Kuščer I. // Physica. 1983. V. 121A. №2. P. 430-456.

[3] Mason E. A., Masur E. // Physica. 1985. V. 130A. №2. P. 437-464.

[4] Пальцев Л. А. // ТМФ. 1988. Т. 77. №3. С. 412-425.

[5] Пальцев Л. А. // ТМФ. 1989. Т. 78. №1. С. 81-93.

[6] Боголюбов Н. Н. Избранные труды в трех томах. Том 2. Киев: Наукова думка, 1970.

[7] Ферцигер Джс., Капер Г. Математическая теория процессов переноса в газах. М.: Мир., 1976.

[8] Köhler W.E., Knaap H.F.P., Eggermont G. E. J., 't Hooft G. W. // Z. Naturforsch. 1978. Bd. 33a. № 7. S. 761-777.

[9] Ландау Л. Д., Лифиии, Е. М. Квантовая механика (нерелятивистская теория). М.: Наука., 1989.

[10] Гольдбергер М., Ватсон К. Теория столкновений. М.: Мир, 1967.

Центральный аэрогидродинамический институт им. Н. Е. Жуковского

Поступила в редакцию 16.VIII.1995 г.

\section{A. Paltsev \\ ON NONEQUILIBRIUM PROCESSES IN MODERATELY DENSE POLYATOMIC GAS IN EXTERNAL MAGNETIC FIELD}

The dissipative and relaxation processes in moderately dense polyatomic gas in external magnetic field are considered by using quantum kinetic theory methods. The momentum and energy fluxes and transport coefficients are obtained in dissipative Navier-Stokes approximation taking into account the first virial correction. The tensor coefficient of shift viscosity in gas of diamagnetic molecules is obtained as a function of binary and ternary effective cross sections and a value of the magnetic field. The Bloch equation for magnetization in space homogeneous gas of linear diamagnetic molecules is derived. Relaxation times and the shift of Larmor's frequency due to intermolecular collisions are obtained. 